\title{
Chromaticity Measurement Via the Fourier Spectrum of Transverse Oscillations
}

\author{
Xi Yang \\ Fermi National Accelerator Laboratory \\ Box 500, Batavia IL 60510
}

\begin{abstract}
Turn-by-turn data from a single BPM includes information on the chromaticity in sidebands displaced above and below the betatron frequency by an amount of the synchrotron frequency. It may be necessary to induce small amplitude synchrotron oscillation by giving the beam a small kick. Power spectrum of the BPM data gives clear chromatic sidebands, and they can be applied to the chromaticity measurement in the Fermilab Booster.
\end{abstract}

\section{Introduction}

The method that has been applied to chromaticity measurement in the Booster relies on knowledge of the ideal lattice, such as dispersion at the BPM location, for the momentum-offset calculation from the orbit data. One can only expect an approximate result from such a measurement, because the maximum discrepancy between the calculated dispersion under operational conditions using MAD and the dispersion from the ideal lattice is more than $20 \%$. A different approach for measuring the chromaticity has been simulated under operational conditions. The sidebands due to the chromatic modulation of the betatron tune through the synchrotron motion are observed, and the chromaticity can be extracted from the characteristic of those sidebands. Also, requirements for such a measurement are described, and they can be applied for the future experiment. 


\section{Method}

A BPM reading $\left(x_{n}\right)$ of a single bunch at the $n^{\text {th }}$ turn after the beam has been excited both transversely and longitudinally can be written as the summation of three different terms:[1]

$x_{n}=x_{c o}+x_{D}(n)+x_{\beta}(n)$.

$x_{c o}$ represents the close orbit and $x_{D}$ represents the displacement due to the dispersion $\left(D_{x}\right)$ :

$x_{D}(n)=D_{x} \times \delta \times \cos \left(2 \pi Q_{s} n+\phi_{0}\right)$.

$Q_{s}$ is the synchrotron tune, and $\phi_{0}$ is the phase of the synchrotron motion at the BPM right after the excitation, which can be set to zero since the phase advance of the synchrotron motion is small within one Booster turn. $\delta$ is

$\delta=\Delta p / p$

And $x_{\beta}$ is the displacement due to the betatron motion:

$x_{\beta}(n)=\hat{x} \times \cos \left(2 \pi Q_{x} n+\frac{\xi_{x} \times \delta \times \sin \left(2 \pi Q_{s} n+\phi_{0}\right)}{Q s}+\phi_{0}^{\prime}\right)$.

$\hat{x}$ is the amplitude of the betatron motion at the BPM, $Q_{x}$ is the betatron tune, and $\xi_{x}$ is the chromaticity. $\phi_{0}^{\prime}$ is the phase of the betatron motion at the BPM on the $0^{\text {th }}$ turn; it depends upon the phase advance between the place where the betatron motion is excited and the place where the BPM is. Here, we set it to zero.

The momentum offset doesn't stay as a constant, and it is changing as $\delta \times \cos \left(2 \pi Q_{s} n\right)$ after the synchrotron motion is excited. The betatron tune is modulated through the chromatic effect, and the betatron tune at time $t$ after the longitudinal excitation can be written as

$\omega_{\beta}(t)=2 \pi\left(Q_{x}+\xi_{x} \times \delta \times \cos \left(2 \pi Q_{s} t\right)\right)$.

The betatron phase $\phi_{\beta}(T)$ at time $T$ is 


$$
\begin{aligned}
& \phi_{\beta}(T)=\int_{0}^{T} \omega_{\beta}(t) d t \\
& =\int_{0}^{T} 2 \pi\left(Q_{x}+\xi_{x} \times \delta \times \cos \left(2 \pi Q_{s} t\right)\right) d t \\
& =2 \pi Q_{x} T+2 \pi \times \xi_{x} \times \delta \times \int_{0}^{T} \cos \left(2 \pi Q_{s} t\right) d t \\
& =2 \pi Q_{x} T+2 \pi \times \xi_{x} \times \delta \times \frac{\sin \left(2 \pi Q_{s} T\right)}{2 \pi Q_{s}} \\
& =2 \pi Q_{x} T+\frac{\xi_{x} \times \delta \times \sin \left(2 \pi Q_{s} T\right)}{Q_{s}} .
\end{aligned}
$$

Finally, we obtain the transverse displacement due to the betatron motion (eq. 4) by substituting $T$ with the turn number $n$ in the betatron phase, as shown by eq. 6 . Here, all the equations are valid in both the horizontal $(x)$ and vertical $(y)$ directions.

\section{Simulation}

The operational parameters were used in the MATLAB calculation. They are the synchrotron tune $Q_{s}=0.08$, horizontal betatron tune $Q_{x}=6.7$, horizontal dispersion in the long straight section $D_{x}=1.6 \mathrm{~m}$, closed orbit $x_{c o}=1 \mathrm{~mm}$, amplitude of the betatron motion $\hat{x}=1 \mathrm{~mm}$, and the number of tracking turns $n=256$. The simulation was performed at two different momentum offsets $\delta=0.0025$ and $\delta=0.005$. In each calculation, the chromaticity was changed from -6 to 6 . For the momentum offset $\delta=0.0025$, the simulated BPM data are shown in Fig. 1(a), and their unnormalized power spectrums are shown in Fig. 1(b). It is hard to see the differences among the different chromaticity settings from the BPM data directly. However, there are clear differences in their power spectrums, as shown in Fig. 1(b). The first peak at 0.08 is caused by the second term of eq.1. The peak value is determined by the product of the dispersion and momentum offset, but independent of the chromaticity. It provides a possibility to measure the dispersion at the BPM position. The peak value of the betatron-tune line is determined only by the amplitude of the betatron motion. Peaks with a separation of synchrotron tune to the betatron-tune line have their peak values, which are determined by the product of the chromaticity and the momentum offset. For the momentum offset $\delta=0.005$, the simulated BPM data are shown in Fig. 2(a), and their unnormalized power spectrums are shown in Fig. 2(b). When the chromaticity is set to 0.2 for the momentum offset $\delta=0.005$, the chromaticity sidebands are still observable, as shown in Fig. 3. Finally, the chromaticity can be 
extracted from the optimal curve fit for the power spectrum of the experimental data by varying chromaticity settings.

\section{Discussion}

This measurement requires both longitudinal and transverse excitations of the beam, and it can be done since both the transverse and longitudinal kickers work well. One more requirement is to turn the radial feedback off during the measurement since the radial feedback tries to suppress the synchrotron motion by correcting the momentum offset of the beam.

\section{Acknowledgement}

The author thanks Dr. Frank Zimmerman for introducing this problem at the course of Beam Control and Manipulation in the UPAS summer school of 2004. Also thanks for the guidance and support from James MacLachlan during this work.

\section{Reference}

[1] M. G. Minty and F. Zimmermann, "Measurement and Control of Charged Particle Beams”, Springer 2003. 


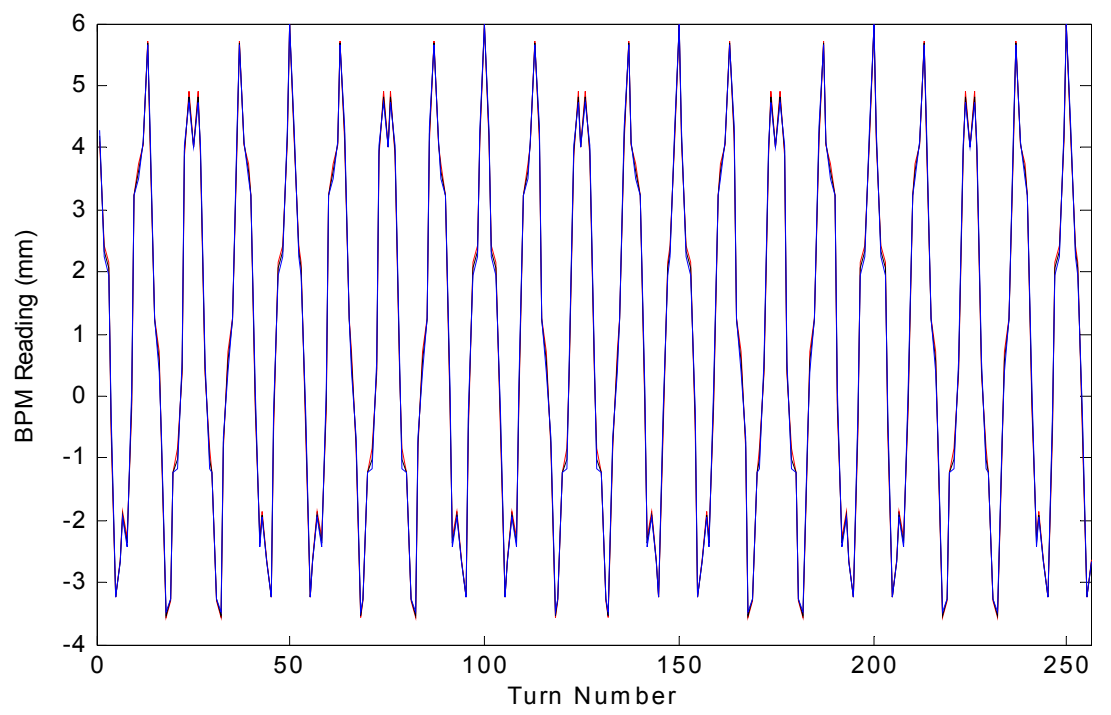

Fig. 1(a)

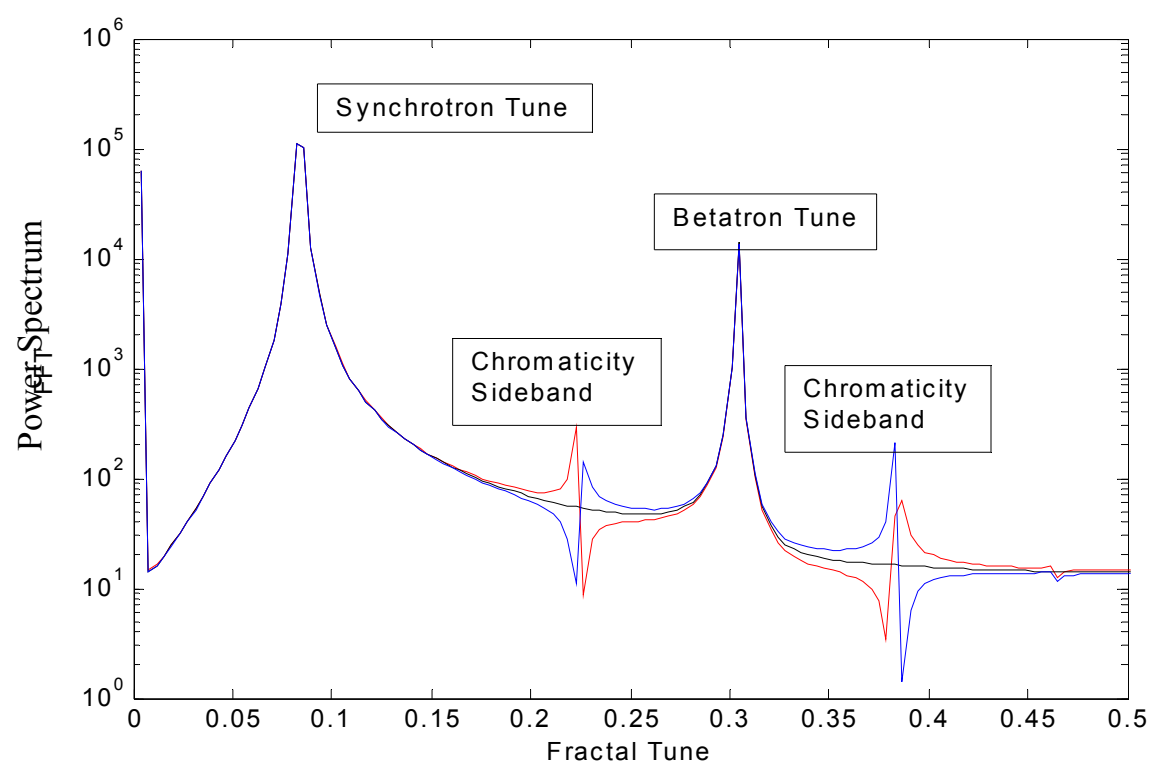

Fig. 1(b)

Fig. 1(a) the momentum offset at $\delta=0.0025$. The BPM turn-by-turn readings were calculated at three different chromaticity values, $-6,0,6$, and they correspond to the red, black and blue curves.

Fig. 1(b) the unnormalized power spectrum of the calculated BPM data in Fig. 1(a). 


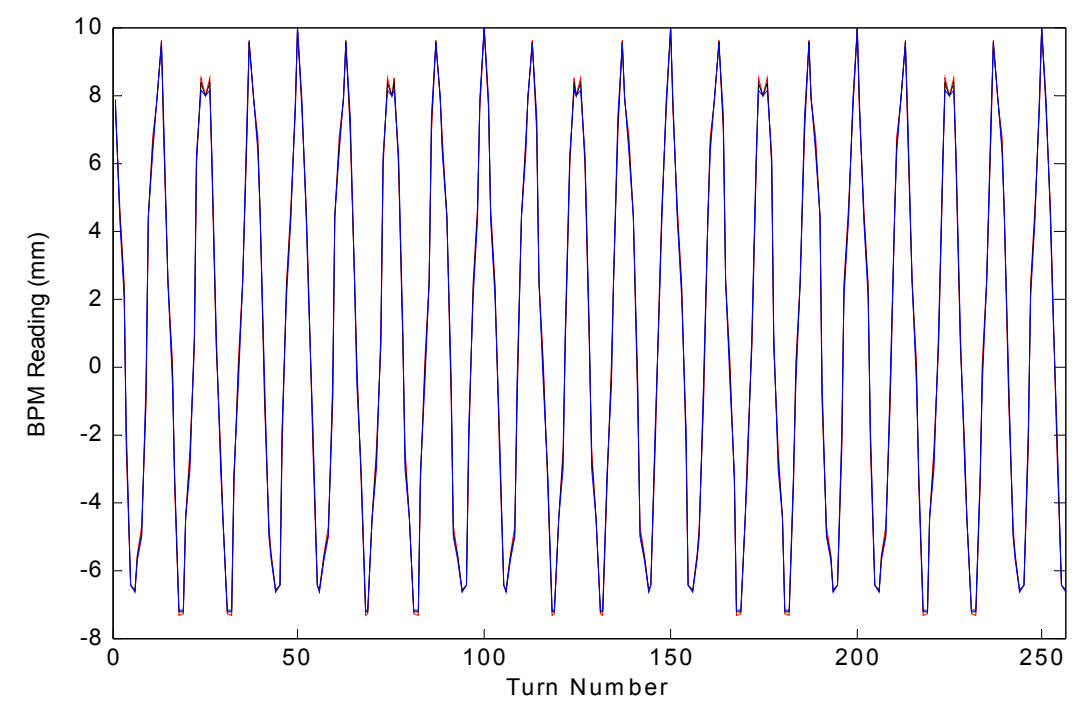

Fig. 2(a)

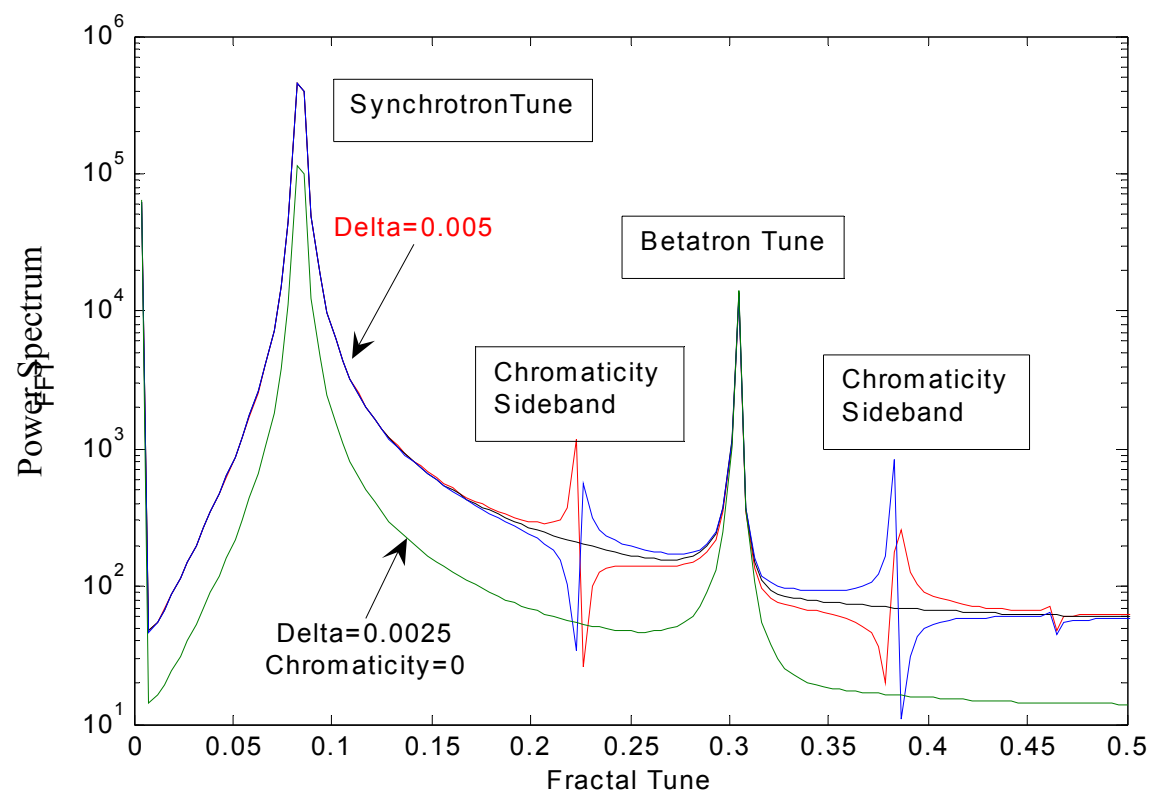

Fig. 2(b)

Fig. 2(a) the momentum offset at $\delta=0.005$. The BPM turn-by-turn readings were calculated at three different chromaticity values, $-6,0,6$, and they correspond to the red, black and blue curves.

Fig. 2(b) the unnormalized power spectrum of the calculated BPM data in Fig. 2(a). The green curve is the same with the black curve in Fig. 1(b). Here, the label of Delta in the graph is equivalent to $\delta$. 


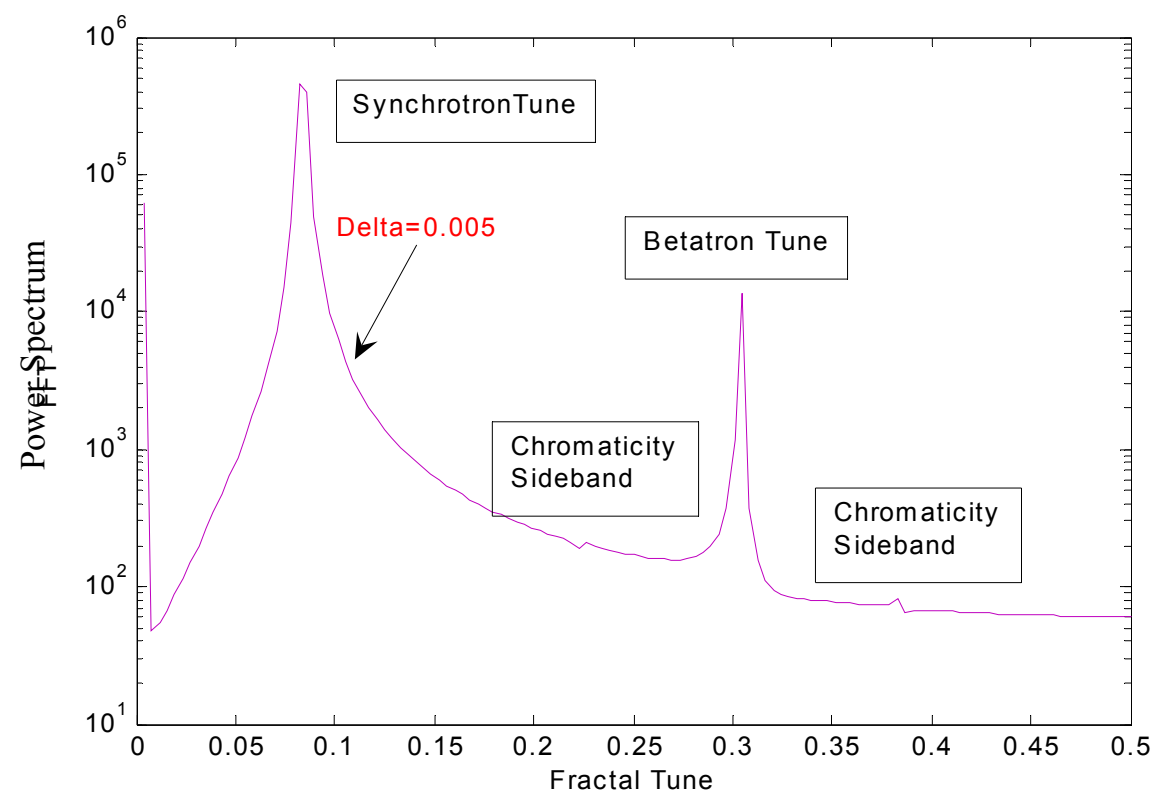

Fig. 3

Fig. 3 the unnormalized power spectrum of the calculated BPM data when the momentum offset at $\delta=0.005$ and the chromaticity at 0.2 . 